\title{
Direct Visualization of the Grain Boundary Solute Segregation in Oxide Material at Atomic Resolution Using STEM-EDS
}

\author{
Bin Feng ${ }^{1}$, Tatsuya Yokoi $^{2}$, Akihito Kumamoto ${ }^{1}$, Masato Yoshiya ${ }^{2,3}$, Yuichi Ikuhara ${ }^{1,3,4}$ and \\ Naoya Shibata ${ }^{1}$ \\ 1. Institution of Engineering Innovation, The University of Tokyo, Tokyo, 113-8656 Japan. \\ 2. Department of Adaptive Machine System, Osaka University, Osaka 565-0871, Japan, \\ 3. Nanostructures Research Laboratory, Japan Fine Ceramic Center, Nagoya, 456-8587, Japan. \\ 4. WPI-AIMR Research Center, Tohoku University, Sendai, 980-8577, Japan.
}

Grain boundaries usually govern the macroscopic properties of polycrystalline materials in practical use. Grain boundary segregation is a fundamental yet critical issue in this field since it affects the local structure and chemistry. Although the advanced aberration-corrected scanning transmission electron microscopy (STEM) has provided deep insights into such complex phenomenon even down to the atomic dimensions [1], most of these studies have been focused on the non-solute segregation. For this sort of segregation, the dopant atoms can only reside at grain boundaries and thus the experimental identification is straightforward [2]. On the other hand, for solute segregation, in which the dopant atoms can reside both at the grain interiors and grain boundaries, it is still challenging to clarify such phenomenon from experiment especially at atomic resolution.

Y segregation in yttria-stabilized zirconia (YSZ) grain boundary is a typical example of grain boundary solute segregation. Such local chemical inhomogeneity has been recognized as the origin of the high grain-boundary resistivity in ionic conductivity [3]. Probing the grain boundary solute segregation at atomic scale is therefore critical for a basic understanding of the chemistry at such interfaces, which in turn will provide new strategies for the design of high performance materials. Here, the local $Y$ distributions near YSZ grain boundaries were directly determined by atomic-resolution elemental mapping using STEM energy-dispersive X-ray spectroscopy (EDS), equipped with a high-sensitivity silicon drift detector (SDD).

In this study, we selected $\Sigma 3[110] /(111)$ YSZ grain boundary as a model grain boundary. YSZ (10 mol\% $\mathrm{Y}_{2} \mathrm{O}_{3}$ doped $\mathrm{ZrO}_{2}$ ) bicrystal containing a $\Sigma 3[110] /(111)$ model $\mathrm{GB}$ was first fabricated by diffusion bonding of two single crystals at 1600 degree for $15 \mathrm{~h}$ [2]. EDS mapping was performed using a JEOL JEM-ARM200CF cold FEG. The accelerating voltage was $200 \mathrm{kV}$. The probe size is about 1.2 $\AA$ with the probe current of about 60 pA. The STEM-EDS system is equipped with double SDD-EDS detectors and the solid angle for the whole collection system is about $1.7 \mathrm{sr}$. Element maps were extracted with $\mathrm{Zr} \mathrm{K}$ line $\left(\mathrm{K}_{\alpha}\right.$ of $15.776 \mathrm{keV}$ and $\mathrm{K}_{\beta}$ of $\left.17.668 \mathrm{keV}\right)$ and $\mathrm{Y} \mathrm{K}$ line $\left(\mathrm{K}_{\alpha}\right.$ of $14.958 \mathrm{keV}$ and $\mathrm{K}_{\beta}$ of $\left.16.738 \mathrm{keV}\right)$.

Figure 1 is a high angular annular dark-field (HAADF) STEM image of the $\Sigma 3[110] /(111)$ grain boundary (indicated by an arrow) from [110] direction. The grain boundary was bonded well at atomic level without any amorphous or secondary phase. Since the atomic column intensity in HAADF STEM image is approximately proportional to the $\mathrm{Z}^{2}$ (where $\mathrm{Z}$ is the atomic number), the bright contrast represent the cation columns intermixed by $\mathrm{Zr}(\mathrm{Zr}=40)$ and $\mathrm{Y}(\mathrm{Y}=39)$. However, it is impossible to differentiate $\mathrm{Y}$ from $\mathrm{Zr}$ atoms from the HAADF STEM image contrast. Hereafter, STEM-EDS mappings were performed at the grain boundary. Figure 2 is the EDS profile obtained from the whole 
STEM EDS map. Figure 3a and d show atomic-resolution STEM-EDS mapping for Zr and Y, respectively. It can be seen that $\mathrm{Y}$ solute atoms strongly segregate in the two layers adjacent to the grain boundary mirror plane. Meanwhile, the $\mathrm{Zr}$ intensities in these two layers are decreased. Our further analysis show that a characteristic ordered segregation occurred, and such segregation structure is energetically favorable [4]. Details will be given in the presentation. [5]

\section{References}

[1] Y. Ikuhara, Journal of Electron Microscopy 60 (2011) p. 173.

[2] N. Shibata et al, Nature Mater 8 (2009) p. 654.

[3] X. Guo and R. Waser, Progress in Materials Science 51 (2006) p. 151.

[4] B. Feng et al, submitted.

[5] This work is supported by: Grant-in-Aid for Scientific Research on Innovative Areas "Nano Informatics" (Grant No. 25106003) from Japan Society for the Promotion of Science (JSPS) and "Nanotechnology Platform" (Project No. 12024046) of the Ministry of Education, Culture, Sports, Science and Technology (MEXT), Japan, Grants-in-Aid for Scientific Research (A) (15H02290) and Grant-in-Aid for Scientific Research (B) (26289234) from the JSPS.

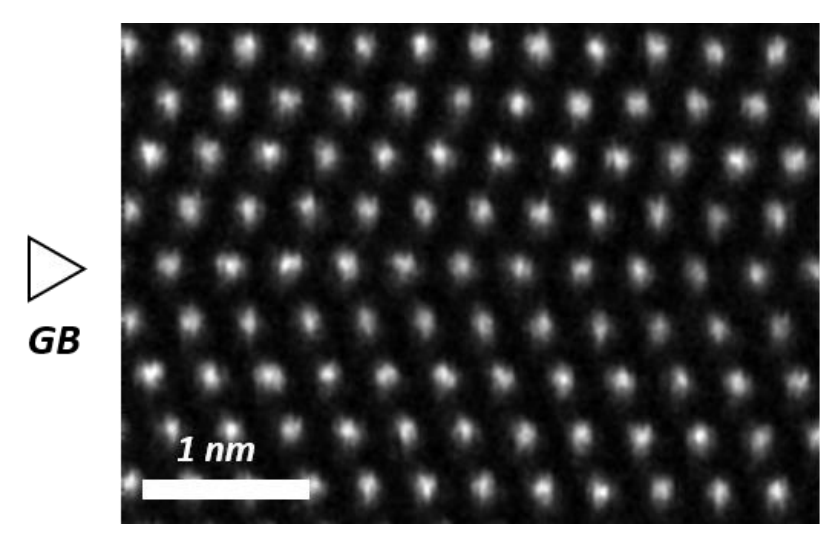

Figure 1. HAADF-STEM image of the $\Sigma 3$ grain boundary

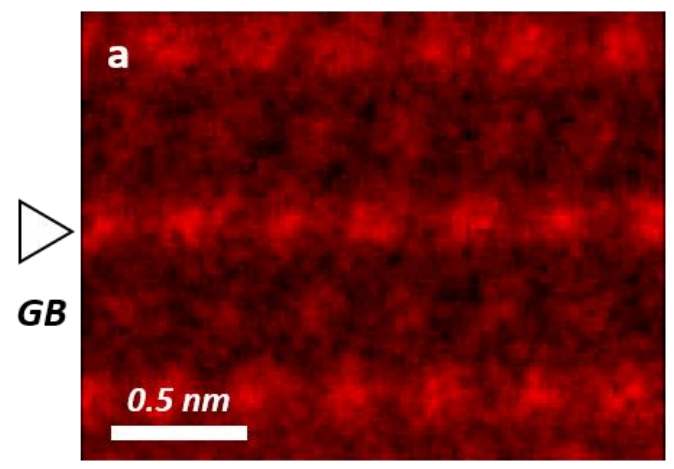

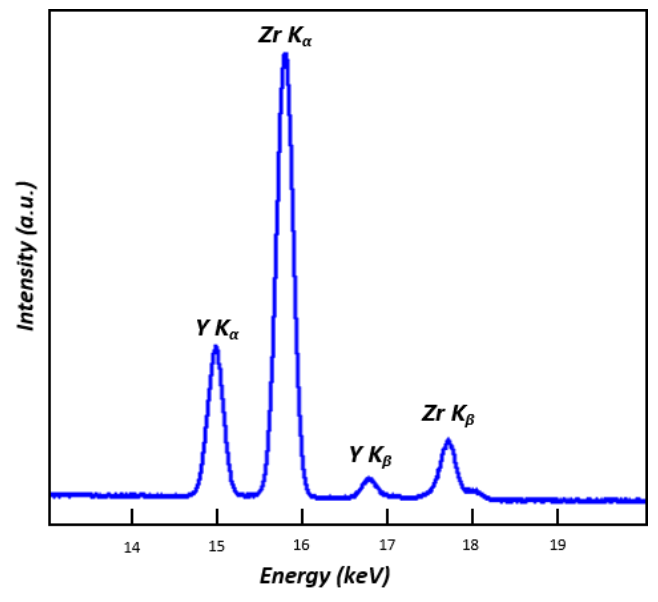

Figure 2. EDS profile extracted from the mapping

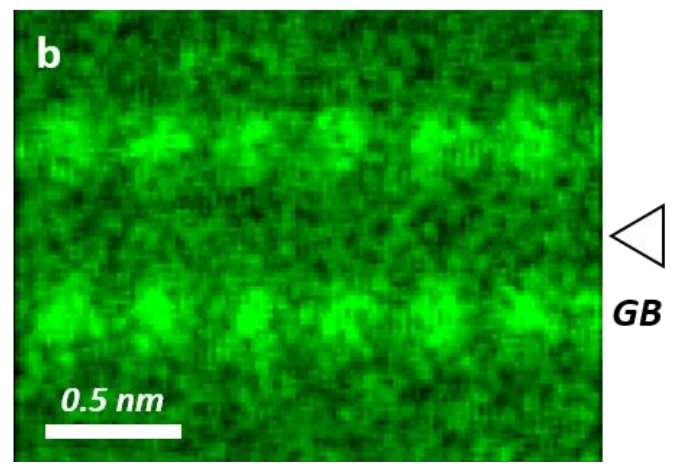

Figure 3. Atomic resolution STEM EDS maps at the grain boundary. (a) Zr map and (b) Y map. 